\title{
Back to Groin; Re-Knocking the Door...
}

\author{
HAMED KADRY, M.D.; OMAR EL SHARKAWY, M.D.; NADEEN EL ESSAWY, M.Sc. and \\ AHMED A. TAHA, M.D.
}

The Department of Plastic \& Reconstructive Surgery, Faculty of Medicine, Cairo University

\begin{abstract}
Background: Old but solid gold. This is the first thing that comes to our mind when we hear the word "Groin Flap".

The inimitable flap holds the advantages of being easily harvested with a relatively concealed donor site and very low morbidity.

Recently, the goals of reconstruction have rapidly progressed from merely filling a hole to providing an improved function, pleasing aesthetic outcome and minimal to no donor site morbidity. Compared to the bulky conventional groin flap, the Superficial Circumflex Iliac artery Flap perforator (SCIP) fulfills the later requirements by being an already thin flap upon harvest, that doesn't require further de-bulking.
\end{abstract}

This yields thin, pliable tissue with a well-concealed donor site scar and minimal donor site morbidity.

In the current study, we represent our experience in using the SCIP flap for various defects of the hand due to different etiologies.

Methods: Twenty SCIP flaps were raised superficial to the breaching fascia to cover various defects of the hand and distal forearm during the period of June 2018 to June 2019. Accordingly, with the preservation of the fascia, the flap relies wholly on the superficial branch of the superficial circumflex iliac artery, along with the sub-dermal plexus, sparing the deep branch.

Results: Twenty patients (14 males and 6 females) with various injuries to the hand were treated with supra-fascial groin SCIP flap.

- The mean age was 26.9 years (range 8-50 years, SD 10.70). - Mean BMI was $27.56 \mathrm{Kg} / \mathrm{m}^{2}\left(20-30 \mathrm{Kg} / \mathrm{m}^{2}\right.$, SD 5).

Supra-fascial flaps were taken as large as $(10 \mathrm{~cm} \times 20 \mathrm{~cm})$. Mean operative time was 3.05 hours $( \pm 0.83)$, and mean followup was eight months (6-12 months, SD 2.1).

All injuries did not require further flap thinning or refashioning.

Conclusion: SCIP flap with its supra-fascial dissection can be safely used as an alternative to the conventional groin flap. With its thin nature, better flap in-setting is obtained, the aesthetic outcome is more pleasant and the need for further revisionary debulking surgeries is almost eliminated.

Key Words: Thin - Perforator - Hand defect - Suprafascial - Debulking.

\section{INTRODUCTION}

With the rapid development of transportation and modern industries, there is an increasing incidence of limb injuries caused by high energy trauma, such as road traffic and machine injuries. As a consequence of the development of surgical repair and reconstruction technology, limbs damaged by high energy trauma that might have needed to be amputated in the past can now be salvaged by surgical reconstruction. This, in turn, demands rapid progression in soft tissue reconstruction to offer patients the best functional outcome along with short recovery time and of course, improved aesthetic outcome [1].

The groin flap, supplied by the (SCIA) has been known for the past decades for its importance, being a paramount flap used for coverage of the hand defects in which there is destruction or devitalization of tissues [2].

First introduced by McGregor and Jackson in 1972, the groin flap is considered a workhorse for upper extremity reconstruction, providing reliable soft tissue coverage to almost all hand and distal forearm defects with exposed tendons or bones, which might require tendon transfer or bone reconstruction later on. The fat layer beneath the flap allows for sliding and gliding of tendons, preventing adhesions and providing optimum results, decreasing the need for future surgeries. In addition, it sets the base for future thumb reconstruction in crushing injuries necessitating toe to hand transfer [3].

Although the groin flap has the advantage of being esthetically pleasing, as the donor-site scar can be covered by underwear, disadvantages that include short pedicle lengths and sizes, anatomical variations in the vessel anatomy, bulky flaps in obese patients, and lymphorrhea within donor sites, have been reported. 
Limited size of the flaps and dependent position of the arm were also among the disadvantages recounted [4].

Another alternative would be the suprafascial dissection of the groin flap, a different technique of flap harvest described by Abdelrahman et al., in 2017. The flap was raised entirely superficial to the deep fascia, and based solely on the superficial branch of the SCIA along with the medial dermal plexus. This new technique avoids opening the deep fascia and minimizes the chances of injury to major vessels, nerves and lymphatic channels, and most importantly decreases the unnecessary flap bulk with no statistical difference to flap survival when compared to the conventional groin flap elevation technique [5].

There are multiple methods for de-fatting of the flap suggested by different authors that are discussed later on in this article.

In this study, a novel technique of dissection one fat cell below the dermis was adopted. Though that is a suprafascial dissection yet thinner than Abdelrahman et al., that leaves a lot of bulky fat cells attached to the flap.

\section{PATIENTS AND METHODS}

This prospective study was conducted on 20 patients presenting to Kasr Al-Ainy Plastic and Reconstructive Surgery Department, Cairo University, including both the emergency department and the outpatient clinic. The study was conducted on patients presenting with various injuries of the hand and distal forearm requiring flap coverage during the period of June 2018 to June 2019 after obtaining ethical committee approval of both Plastic Surgery Department as well as the Faculty of Medicine.

\section{Inclusion criteria included patients with:}

- Volar raw areas with exposed tendons/bones.

- Patients requiring bone reconstruction.

- Raw areas requiring flap coverage on wrist and distal forearm.

- Dorsal raw areas with exposed tendons and bones.

- Candidates for later on toe to hand transfer.

- Candidates for later on tendon grafts or transfer.

- Post burn contracted scar on dorsum of hand.

- Previously contracted skin graft on dorsum of hand.

- Finger defects with exposed tendons or bones.

- Patients with contracted scars on the palm.

- Patients with contracted web space.

\section{While Exclusion criteria were:}

- Patients refusing to perform the surgery.

- Pregnancy.

- Smokers.

- Diabetes and hypertension.

- Previous inguinal surgeries.

- Previous irradiation to inguinal area.

- Uncooperative or mentally retarded patients.

- Raw areas caused by excision of malignant lesions.

- Failure of previous flap coverage.

Patients who matched the criteria were consulted initially about the procedure, their possible options, and their willingness to participate in the study. A second consultation was offered to all patients with extra details about the advantages and disadvantages of the technique as well as alternatives. Approval by the department local ethical committee was obtained with verbal and written consent from all patients participated.

All patients presented to the emergency department were scheduled for an initial debridement on the day of admission and intraoperative reassessment \& fixation of any concomitant bony fractures. The patients were reassessed on the $4^{\text {th }}$ day of admission concerning the need for further debridement session or moving on to definitive management. Patients presented to the outpatient clinics were mainly due to hand post burn contracture deformities.

Marking of the important landmarks included: Fig. (1)

1- Anterior superior iliac spine, symphysis pubis, and the inguinal ligament as a line extending between them.

2- The femoral artery was drawn after confirmation of its position by a handheld Doppler, and the origin of the SCIA was marked $2.5 \mathrm{~cm}$ below the femoral artery.

A line was drawn from the origin of SCIA parallel to the inguinal ligament. This line was considered as central axis of the flap on which the flap design was formulated (Fig. 1).

A handheld Doppler (5-8MHz) was used to locate the superficial and deep branches of the SCIA and their perforators. The course of superficial \& deep branches was followed using the Doppler and marked. 
Preparation of the affected upper limb was the first step of surgery either by meticulous debridement in for traumatic cases or by proper excision and release of the scar re-creating the defect in contracture scared cases.

Moving on to flap harvesting, using a 15-scalpel blade, the flap was incised all around as marked. Starting at the inferior and lateral borders, the dermis was incised, beveling outwards away from the flap. Stay sutures using polyglactin $2 / 0$ were taken along the edge to avoid excessive handling of the flap edges using skin hook or toothed forceps. Tedious dissection was started using small, blunt dissection scissors until we reach the desired plane, one fat cell layer below the dermis. The Scarpa's fascia was not included at any point in the flap (Figs. 2,3).

Once this plane was acquired, dissection was completed from lateral to medial and in the cephalic direction until the lateral border of the Sartorius's muscle was visualized.

At this level, dissection was carried on but in a deeper plane, yet above the Sartorius fascia to ensure that the pedicle was not injured during the procedure leaving behind the deep branches. Visualization of the branches SCIA was not an essential part of the procedure as they were anatomically in a different plane.

It is important to note that including the Sartorius fascia in this area did not affect the thickness of the flap as this part was not usually used in the coverage of the raw area, yet it ensured the safety of the pedicles which in turn increased the success rate of the flap.

After reaching the medial border of the Sartorius, the flap was checked for dermal bleeding,

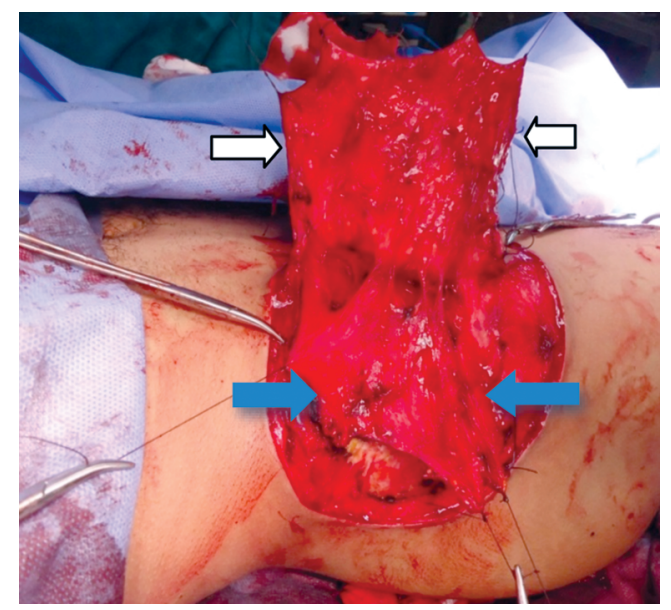

wrapped in warm saline-soaked foments, and was left for a while until hemostasis of the bed was performed.

At this stage, dissection and undermining of the abdomen and thigh were performed as needed to allow primary closure of the donor site under the least possible tension.

The final step in this operation would be the in-setting of the flap. It was noted that the ideal method to do this would be from the ulnar border of the upper limb. This way, the weight of the limb will not cause traction in the opposite direction, which might cause shearing of the flap from its bed.

Flap separation was done three weeks later. Patients were followed-up once weekly for the first month, and monthly thereafter for six months. (Figs. 4,5).

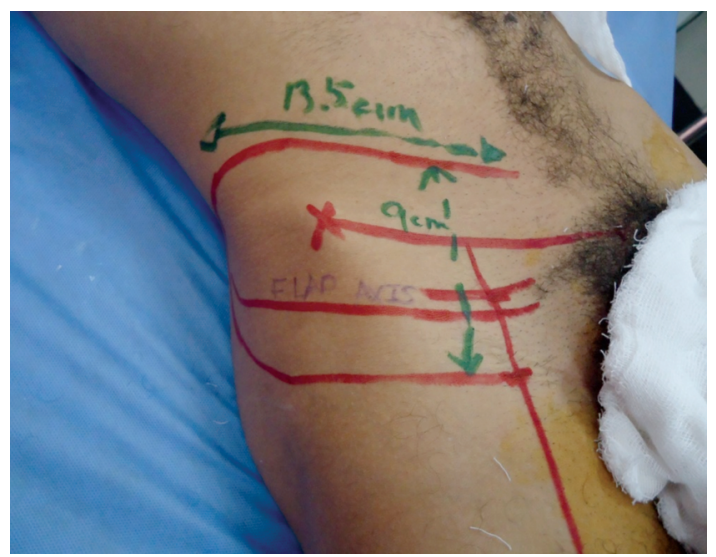

Fig. (1): Marking of the groin area in preparation for SCIA perforator flap. A line was drawn from the origin of SCIA parallel to the inguinal ligament. This line was considered as central axis of the flap on which the flap design was formulated.

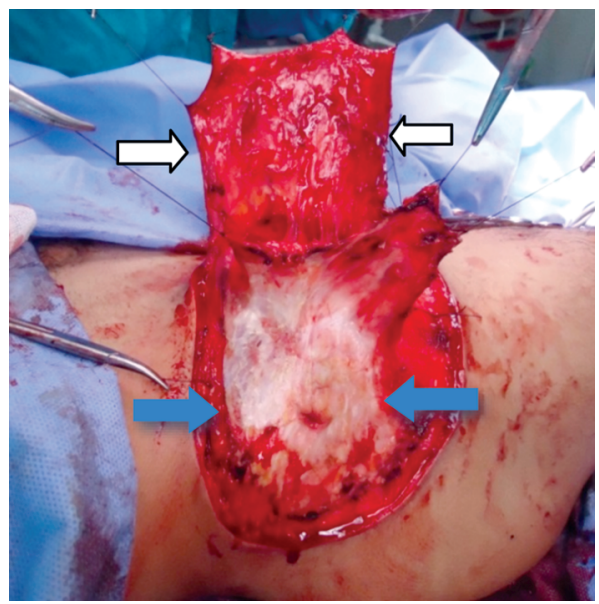

Fig. (2): Tedious Dissection is started until the desired plane was reached; one fat cell layer below the dermis. The Scarpa's fascia was not included at any point to the flap. The white arrows show the flap after dissection, blue arrows show the Scarpa's fascia. 
(A)

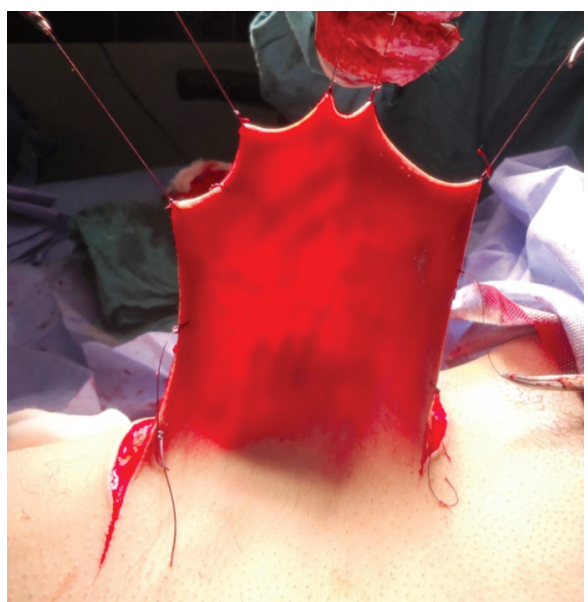

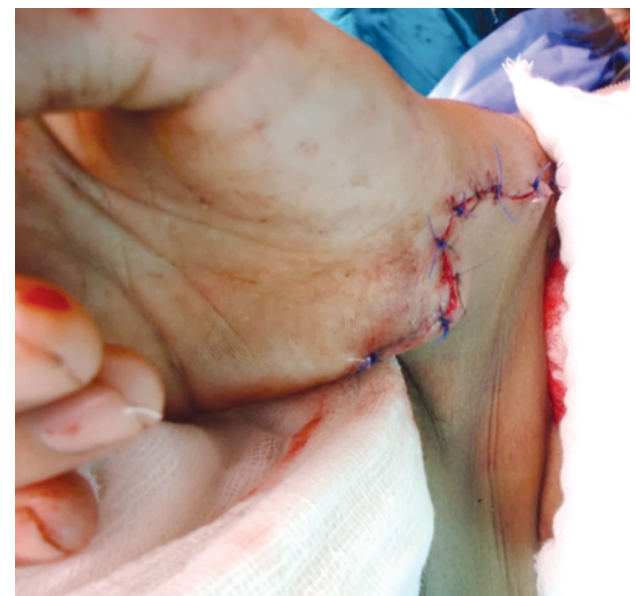

(B)

Fig. (3): (A) Trans-illumination through the flap. (B) Note the skin indentation (red arrow). Both (A) and (B) Denotes the thin nature of the flap.

Fig. (4): (A,B): Pre-operative post burn contracture deformity of the left hand (C) In this patient, the accidental presence of conventional groin flap on the right side led to comparison in the thickness between the conventional groin flap (right hand, white arrow) and the SCIA perforator flap (left hand, red arrow) showing the thickness difference between both hands. (D) (E) These photos were taken 6 months post-operative, right hand with conventional groin flap (below) and left hand with SCIA perforator flap (above) though inadequate release of the MP joints can be noticed.
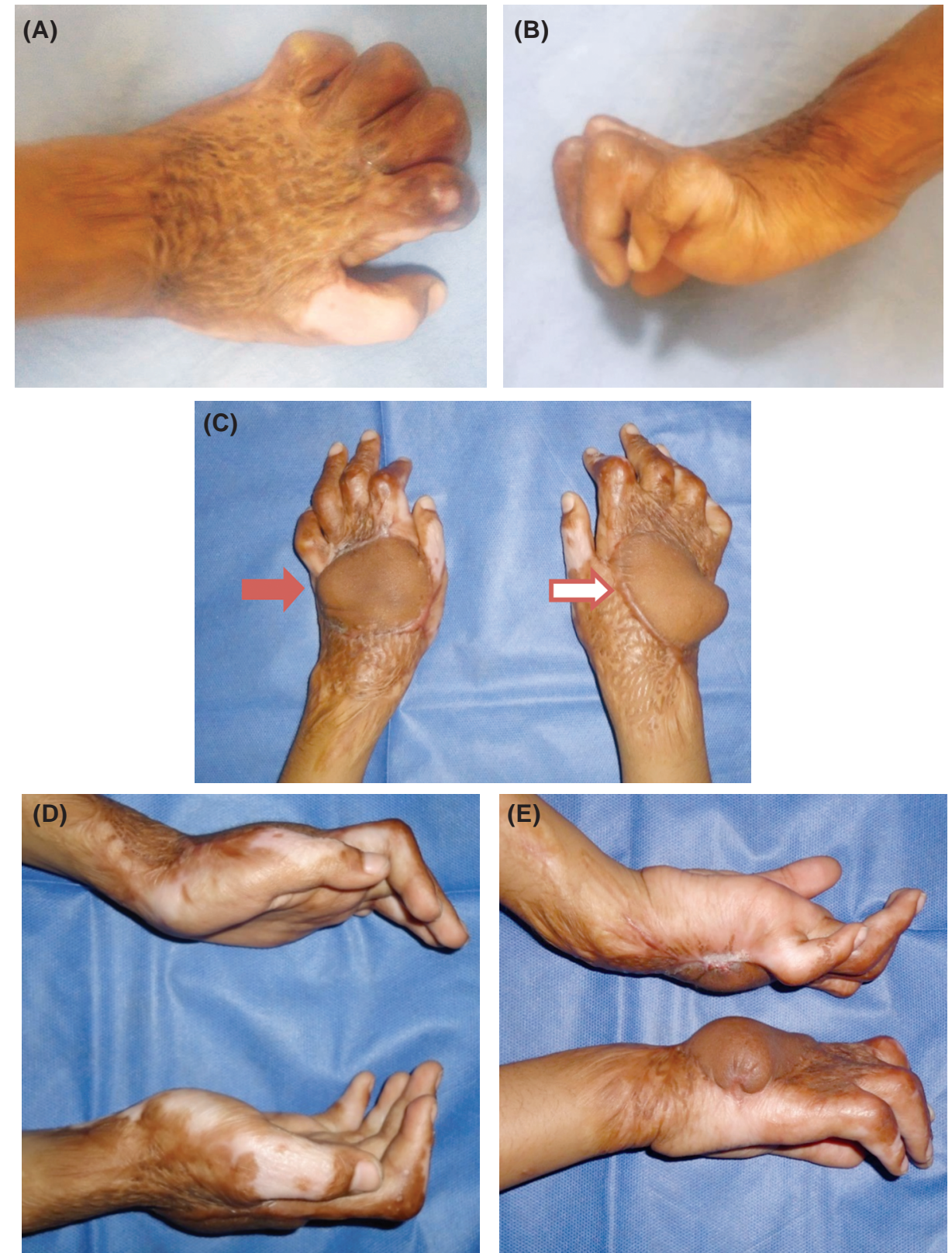
(A)

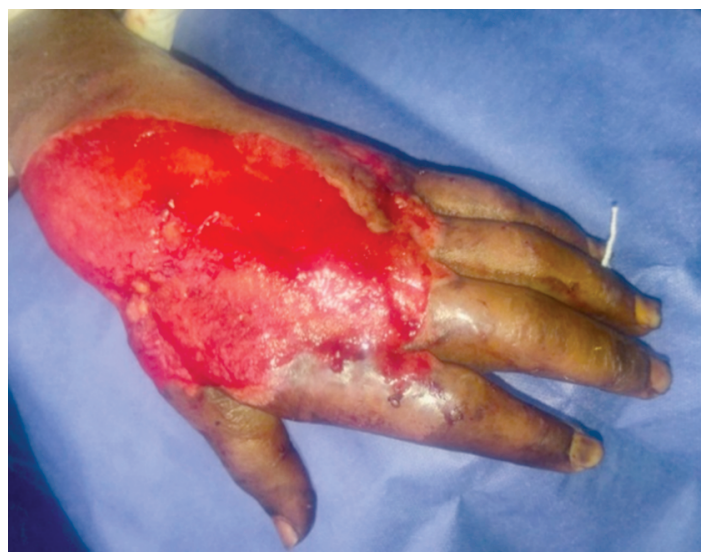

(C)

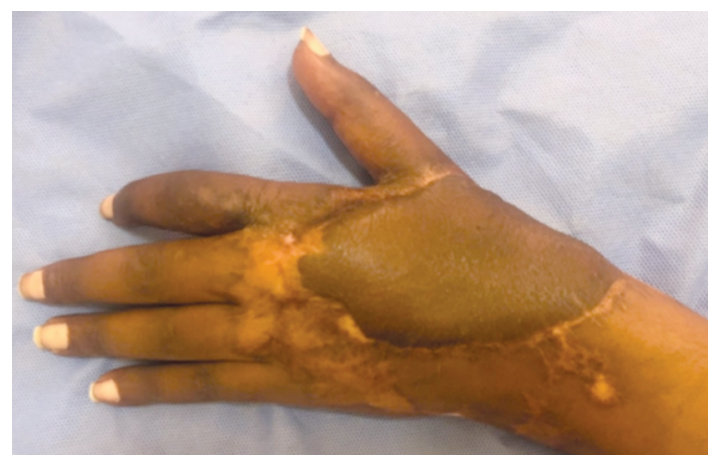

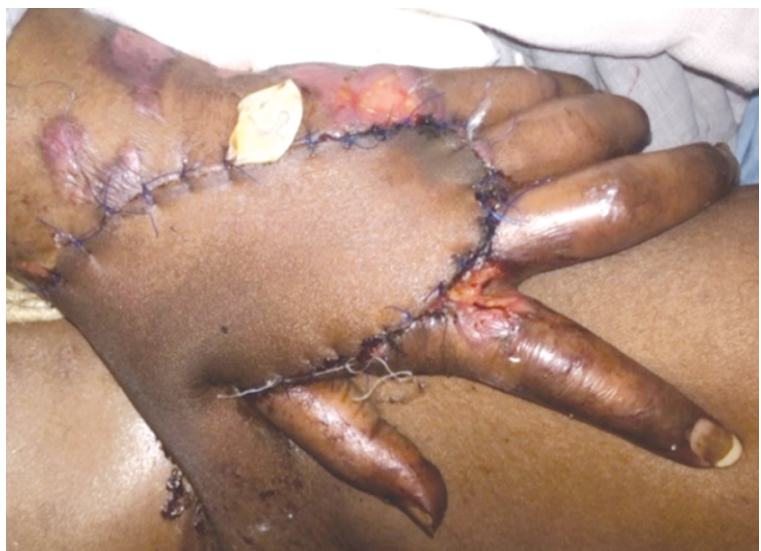

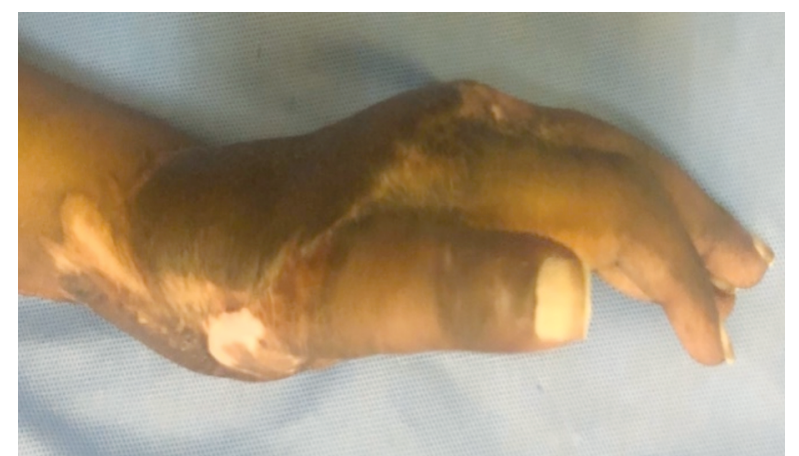

(D)

Fig. (5): (A) Raw area over dorsum of left hand after debridement and vacuum dressing application. (B) SCIA perforator flap after insetting. (C,D) Different views of the hand 6 months post-operative.

\section{RESULTS}

Twenty patients (14 males and six females) with various injuries to the hand were treated with supra-fascial groin SCIP flap. The mean age was 26.9 years (range $8-50$ years, \pm 10.70 ). Mean BMI was $27.56 \mathrm{~kg} / \mathrm{m}^{2}$ (20-30, SD 5). Supra-fascial flaps were taken as large as $(10 \mathrm{~cm} \times 20 \mathrm{~cm})$. Mean operative time was 3.05 hours $( \pm 0.83)$, and the mean follows-up was eight months (6-12 months, $\pm 2.1)$. All injuries did not require further flap reconstruction.

Eleven cases $(55 \%)$ were due to trauma (crushing injury $30 \%$, contused wounds $20 \%$, and shotgun injury 5\%). The remaining 9 cases (45\%) were due to post-burn contracture. All the patients underwent flap coverage using the ipsilateral side.

Nine out of the 11 traumatic patients required flap coverage for the dorsum of the hand (extensor tendons, exposed metacarpals, or K-wire), and two patients for coverage of exposed ulnar nerve. As for patients with contracture deformities, two flaps were used to cover the flexor tendon, three flaps to cover the FPL tendon, and 4 for coverage of extensor tendons, all of which have been exposed during the release of the contracted scar.
The mean defect size was $(5.9 \times 7.4 \mathrm{~cm}, \pm 2.28)$, least of which was $(3 \mathrm{~cm} \times 5 \mathrm{~cm})$ while largest was $(10 \mathrm{~cm} \times 20 \mathrm{~cm})$. The mean flap size was $(7.68 \mathrm{x}$ $10.73 \mathrm{~cm}, \pm 1.82)$. The smallest flap raised was $(5 \mathrm{~cm} \mathrm{x} 7 \mathrm{~cm})$, while the largest flap was $(10 \mathrm{~cm} \mathrm{x}$ $23 \mathrm{~cm}$ ). The mean operative time was 3 hours (SD 0.83 not showing any relation with the rate of complications with $p$-values $<0.37$.

Complications were divided into two groups: complications of the flap and those of the donor site. Of all the study groups, only three patients encountered post-operative flap complications $(10 \%)$, one was total flap necrosis, and the other two were flap avulsion. The patient who experienced flap necrosis had an uncomplicated operative course, with normal flap color, temperature, and dermal bleeding at the time of insetting. However, 12 hours later, edema and color change of the flap took place, starting distally at the periphery and extending progressively to the central part of the flap.

Nitroglycerine showed a lot of promising results in multiple studies [6]. It was applied over the flap in a trial to enhance the arterial supply to the flap but with no significant effect.

The other complication was a patient who experienced flap avulsion during an epileptic fit 1- 
week post-operative, to whom re-insetting was done immediately after control of his neurologic condition. Another patient experienced flap avulsion from the recipient site during recovery from general anesthesia, and the flap was immediately sutured back in place. Both patients did not experience any complications with the flap vascularity, and they had a smooth recovery. The overall flap survival rate was $(95 \%)$.

Regarding the donor site morbidity, three patients had dehiscence of the donor site; the BMI of all was $\geq 30$. None of them required re-operation or closure of the wound with secondary sutures. All of them were treated conservatively by repeated dressings twice daily using an antiseptic solution.

The relationship between BMI and rate of occurrence of donor site morbidity was regarded as statistically significant ( $p$-value $<0.046$ ).

\section{DISCUSSION}

Over the last few decades with with the rapid rhythm of life and everybody running from one place to the other, complex wounds of the upper extremities are becoming more and more common. Those are due to various etiologies including and not limited to crushing, avulsion, road traffic and work related injuries.

These problematic wounds usually involve multiple components including, skin, bone, tendon, and neurovascular structures [7].

The new philosophy of skin coverage should include not only replacing the missing component but also preservation of the function of the hand. The ideal coverage method though not always available should be stable, durable, allow free joint mobility, and should have an aesthetically pleasing with minimal donor site morbidity [8].

Since 1972, the groin flap underwent a lot of studies to formulate a better understanding of its uses, reliability and variability of its vascular anatomy.

The groin flap has the advantage of being a reliable $\&$ versatile flap for complex hand $\&$ forearm defects. As a pedicled flap, it is easy \& fast to harvest with great outcome and easy to learn and to teach [5].

While as a free flap, it lacks most of those advantages as it needs a more advanced microsurgical setting, long operative time and short pedicle makes the anastomosis more challenging [9].
Although the radial or the reversed radial forearm flap are a good alternative to the groin flap providing a thin skin paddle yet it comes at a very high cost (visible donor site, giving up the radial artery); the same goes for posterior interosseous and reversed ulnar artery forearm flap. The anterolateral thigh solved the problem of the visible or ugly donor site with a big skin paddle(especially in females). Yet it is usually bulking requiring a secondary debulking procedure.

The advantages of both the radial forearm \& anterolateral flaps can now be combined in a single flap the superficial circumflex iliac artery perforator flap. It provides a thin pliable flap with a hidden donor site \& low donor site morbidity without the drawbacks of the other flaps [10].

With the rise of the perforator flap era, Koshima revived and revisited the groin flap giving birth to a new flap called the superficial circumflex iliac artery perforator (SCIP) flap in 2004 [11]. The SCIP flap yields thin, pliable tissue with a well-concealed donor site scar and minimal donor site morbidity, which is like that of harvesting a full-thickness skin graft from the groin [12].

Twenty patients were included in this study. In correlation to other studies, 30 patients were performed by Kimura [13], five patients by Green [14], six by Choi [15], 41 by Lee [16], and 28 by Abdelrahman [5]. The largest study group was 210 by Goh. However, only 19 of the patients underwent flap coverage for upper extremity reconstruction [10].

In this study, cases were mainly due to trauma $(55 \%)$ and post burns contracture deformities $(45 \%)$. Cases following tumor resection were not included.

It is, nonetheless, of crucial importance to state that neither of the studies above mentions any statistical significance between the mode of trauma and the rate of flap complications. Most of the literature discussed the plane of dissection of the free SCIP flap; as a result, it is important to note that most of the flap complications recorded were due to either arterial or venous insufficiency.

In this study, the average flap dimensions were $(7.6 x 10 \mathrm{~cm})$, only 1 out of the 20 cases was exceptionally large with dimensions of $(10 \times 20 \mathrm{~cm})$. Average flap dimensions of Kimura, Choi, Goh, Lee and Abdulrahman were $(2 \times 5-12 \times 28 \mathrm{~cm}),\left(75.6 \mathrm{~cm}^{2}\right)$, $(3.5 \times 5-12 \times 25 \mathrm{~cm}),\left(16.2 \mathrm{~cm}^{2}\right)$ and $(9 \times 20 \mathrm{~cm}) \mathrm{re}-$ spectively. In the study by Green, no flap dimensions were mentioned $[\mathbf{5 , 1 0 , 1 4 - 1 6 ]}$. It is hereby 
important to note that the maximum safe dimensions of the conventional groin flap stated in literature are $(17 \times 27 \mathrm{~cm})[13]$.

One method of flap harvest presented by Kimura is to be "elevated in a thin layer of adipose tissue after microdissection of the perforator from the deep branch of the superficial circumflex iliac system (SCIS)". Nevertheless, having a homogenous thin flap in one elevation procedure is not always achieved. It is very difficult to elevate a uniformly thin, large flap in a single-flap-elevation procedure. In that case, the so called the 'wormeaten defatting' method for additional thinning of the flap is advised [13].

Moreover, Choi suggested another plane of dissection of the SCIP flap where elevation is made underneath the superficial fascia just beneath the smaller superficial fat and above the larger deep fat lobules. The same plane was described by Goh $[10,15]$.

Lee, however, described a different way of dissection, yielding an even thinner flap. This was through dissecting immediately below the subdermal plane, yielding as flap as thin as possible [16].

The current study combined two of the previous papers to optimize the results given the thickness of the flap and its survival rate. We were guided by Lee's results after raising superficial subcutaneous nearly sub-dermal flaps [16] and Kimura, who performed extra thinning after raising the supra-fascial flap with no evidence of disturbing its vascularity [13]. The flap was raised thin from the start, in the subdermal plane with no need of extra thinning or de-bulking, neither after its elevation nor later in the six months follow-up course of the patients. It's worth mentioning that some of the patients will still need further surgery for refashioning of the flap. This is because most flaps raised at the beginning of this study were designed larger than the defect, as a precaution in case of distal necrosis that it can then be easily re-advanced.

None of the previous complications were thought to be due to age, BMI, or flap dimensions except for what was mentioned by Kimura for the cases of partial flap necrosis [13]. However, it was markedly noted that the planes of dissection vary according to the BMI, where it was easier to identify smaller superficial fat from larger deep fat in candidates with higher BMI. Also, flap elevation in thin individuals can be done directly supra-fascial with no extra thinning, and the results will still be super thin flaps requiring no further debulking.
In this study, three patients experienced minor dehiscence of the donor site that required no surgical intervention, only repeated dressings. All the patients with donor site complications had BMI $>30$. This was a statistically significant relationship with ( $p$-value <0.046).

The difference in the thickness of conventional and supra-fascial groin flap was noticeable in all patients and was significantly evident in those with higher BMI > 26. This was exceptionally obvious with one of the patients in this study who underwent a conventional groin flap to the dorsum of one hand and supra-fascial flap to the other one. This precise patient sets a ground for future comparative studies in this field.

\section{Conclusion:}

The functions of the hand and upper limb need supple and durable skin coverage to withstand the continuous pressure and friction caused by hand usage [17]. Though single staged procedures with early rehabilitation of the hand are usually targeted yet they are not always feasible. That's where the pedicled suprafascial groin flap provides a safe option for coverage of various upper limb defects. It is far superior than conventional flap when it comes to aesthetic, functional outcome, thickness and better donor site scaring while maintaining similar success rates.

Ultrathin flap can be safely raised in the supraScarpa manner to provide the new thin reliable workhorse flap for resurfacing skin defects.

This work yielded the following recommendations:

- The flap can be raised in a very thin almost subdermal plane, yet it was found safer that the dissection continues in full thickness subfascial plane once the operator reaches the lateral border of the Sartorius. This should ensure maximum safety of the pedicle yet does not affect the thickness of the flap as this part is not used in coverage of the defects.

- The flap better be the exact size of the defect or few millimeters larger. Harvesting a flap with an adequate size will provide the patient with suitable coverage that does not require further surgeries aiming at debulking or refashioning of the flap.

Patients with high BMI >30 are more liable to donor site morbidity. It is thus advised to closely monitor the patient for development of seroma, lymphorrhea or dehiscence as early management will prevent progress of the condition. 


\section{REFERENCES}

1- Zeng Q., Cai G., Liu D., Wang K. and Zhang X.: Successful salvage of the upper limb after crush injury requiring nine operations: A case report. Int. Surg., 100 (3): 540-546. doi:10.9738/INTSURG-D-14-00018.1, 2015.

2- Iida T., Mihara M., Yoshimatsu H., Narushima M. and Koshima I.: Versatility of the superficial circumflex iliac artery perforator flap in head and neck reconstruction. Ann. Plast. Surg. Mar., 72 (3): 332-6. doi: 10.1097/ SAP.0b013e318260a3ad, 2014.

3- Neligan P. and Chang J.: Plastic Surgery. Philadelphia: ElSevier Saunders, Volume 1, p 512e1-515, 2012.

4- Kim J., Kim K. and Yoon C.: Reconstruction of ModerateSized Distal Limb Defects using a Superthin Superficial Circumflex Iliac Artery Perforator Flap. Journal of Reconstructive Microsurgery, 31 (09): pp. 631-635, 2015.

5- Abdelrahman M., Zelken J., Huang R., Hsu C., Lin C., Lin Y. and Lin C.: Suprafascial dissection of the pedicled groin flap: A safe and practical approach to flap harvest. Microsurgery, 38 (5): pp. 458-465, 2017.

6- Argas C.R., Iorio M.L. and Lee B.T.: A systematic review of topical vasodilators for the treatment of intraoperative vasospasm in reconstructive microsurgery. Plast. Reconstr. Surg., 136: 411-422, 2015.

7- Bashir M.M., Sohail M. and Shami H.B.: Traumatic Wounds of the Upper Extremity. Hand Clinics, 34 (1): 61-74, 2018.

8- Griffin M., Hindocha S., Malahias M., et al.: Flap decisions and options in soft tissue coverage of the upper limb. Open. Orthop. J., 8: 409-14, 2014.

9- Goertz O., Kapalschinski N., Daigeler A., Hirsch T., Homann H.H., Steinstraesser L., et al.: The Effectiveness of Pedicled Groin Flaps in the Treatment of Hand Defects: Results of 49 Patients. The Journal of Hand Surgery, 37 (10): 2088-2094, 2012.
10- Goh T., Park S., Cho J., Choi J. and Hong J.: The Search for the Ideal Thin Skin Flap. Plastic and Reconstructive Surgery, 135 (2): pp. 592-601, 2015.

11- Koshima I., Nanba Y., Tsutsui T., et al.: Superficial circumflex iliac artery perforator flap for reconstruction of limb defects. Plast. Reconstr. Surg. Jan., 113 (1): 233e40, 2004.

12- Feng S., Xi W., Zhang Z., Tremp M., Schaefer D., Sadigh P., Zhang W. and Zhang Y.: A reappraisal of the surgical planning of the superficial circumflex iliac artery perforator flap. Journal of Plastic, Reconstructive \& Aesthetic Surgery, 70 (4): pp. 469-477, 2017.

13- Kimura N., Saitoh M., Hasumi T., Sumiya N. and Itoh Y.: Clinical application and refinement of the microdissected thin groin flap transfer operation. Journal of Plastic, Reconstructive \& Aesthetic Surgery, 62 (11): pp. 15101516, 2009

14- Green R., Rahman K., Owen S., Paleri V., Adams J., Ahmed O. and Ragbir M.: The superficial circumflex iliac artery perforator flap in intra-oral reconstruction. Journal of Plastic, Reconstructive \& Aesthetic Surgery, 66 (12): pp. 1683-1687, 2013.

15- Choi D., Goh T., Cho J. and Hong J.: Thin Superficial Circumflex Iliac Artery Perforator Flap and Supermicrosurgery Technique for Face Reconstruction. The Journal of Craniofacial Surgery, p. 1, 2014.

16- Lee K., Park B., Kim E., Kim J., Jang K., Choi S., Lee D. and Mun G.: Superthin SCIP Flap for Reconstruction of Subungual Melanoma. Plastic and Reconstructive Surgery, 140 (6): pp. 1278-1289, 2017.

17- Reyad Khaled A. and Tallal Raghda S.: Bilateral Combined Groin and Hypogastric Flap for Coverage of Extremely Huge Combined Hand, Forearm and Elbow Defects Egypt, J. Plast. Reconstr. Surg., Vol. 43, No. 3, October: 545$547,2019$. 